\title{
Lattice Dynamics and the High Pressure Equation of State of Au
}

\author{
C. W. Greeff and M. J. Graf \\ Los Alamos National Laboratory, Los Alamos, NM 87545, USA
}

(Dated: October 30, 2018)

\begin{abstract}
Elastic constants and zone-boundary phonon frequencies of gold are calculated by total energy electronic structure methods to twofold compression. A generalized force constant model is used to interpolate throughout the Brillouin zone and evaluate moments of the phonon distribution. The moments are used to calculate the volume dependence of the Grüneisen parameter in the fcc solid. Using these results with ultrasonic and shock data, we formulate the complete free energy for solid Au. This free energy is given as a set of closed form expressions, which are valid to compressions of at least $V / V_{0}=0.65$ and temperatures up to melting. Beyond this density, the Hugoniot enters the solid-liquid mixed phase region. Effects of shock melting on the Hugoniot are discussed within an approximate model. We compare with proposed standards for the equation of state to pressures of $\sim 200 \mathrm{GPa}$. Our result for the room temperature isotherm is in very good agreement with an earlier standard of Heinz and Jeanloz.
\end{abstract}

\section{INTRODUCTION}

The elastic constants, phonon frequencies, and equation of state (EOS) are fundamental properties of matter. The values of these parameters under compression find application in geophysics 1 and in the prediction and interpretation of processes of dynamic compression. ${ }^{2.3}$ For many materials, especially elemental metals, the principal Hugoniot curves, the set of states accessible via a single shock from ambient conditions, have been measured ${ }^{2.4 .5 .6}$ Since the pressure, density, and internal energy are known along the Hugoniot from the jump conditions, $\frac{2}{3}$ this data is an important baseline for high pressure equations of state. The off-Hugoniot EOS is needed for the prediction of processes involving more complicated loading paths, such as multiple shocks or shock and release ${ }^{7}$ Extrapolation from the Hugoniot has also been used to establish pressure standards for static high pressure experiments, whose room temperature isotherms are regarded as known ${ }^{2.8 .9}$ Au has been used as a standard in this way, and has been used to calibrate the ruby $R_{1}$ line as a secondary standard.9.10.11 Recent studies have called into question the accuracy of the gold pressure standard. Akahama et al $l^{12}$ compressed Au and Pt in the same cell and found two Au standards 8.13 to give pressures lower than Pt by 20 and $15 \mathrm{GPA}$, respectively, at $150 \mathrm{GPa}$. Shim et al., 14 on the other hand, propose a new EOS for $\mathrm{Au}$ that gives pressures still lower than either of these earlier standards.

To relate the Hugoniot to the room temperature isotherm requires information on the Grüneisen parameter $\gamma=V(\partial P / \partial E)_{V}$ and the specific heat $C_{V} \stackrel{2}{2}$ These are dominated by lattice vibrations in the regimes under consideration here. With increasing compression, the separation between the Hugoniot pressure and the room temperature pressure increases, resulting in greater dependence of the inferred room temperature isotherm on $\gamma$. Because $\gamma$ is not easily measured at high pressure, this introduces a nonnegligible source of uncertainty in the pressure standards. In most cases, a model assumption of the form $\gamma(V)=\gamma\left(V_{0}\right)\left(V / V_{0}\right)^{q}$ has been used. The specific value $q=1$ has been used often in shock work ${ }^{2.8}$ However, a power law dependence of $\gamma$ on $V$ is qualitatively incorrect at high compression, and extrapolation on this basis is inherently limited.

In principle, the various components of the EOS - the static lattice energy, the vibrational free energy, and the electronic excitation free energy - can be evaluated from electronic structure theory. Practical calculations based on approximate density functional theories typically have errors of several percent in the density at zero pressure, and $10 \%$ or more in the bulk modulus ${ }^{15}$ These are unacceptably large errors for the purpose of high accuracy equations of state. These properties can be measured accurately, and are mainly determined by the cold energy curve, which is the largest contribution to the EOS in the regime considered here. On the other hand, we find that ab initio electronic structure calculations are capable of obtaining phonon frequencies of sufficient accuracy to strongly constrain the volume dependence of the Grüneisen parameter $\gamma$. We therefore propose that the most accurate EOS in the solid is obtained by combining an empirical cold energy with ab initio lattice vibration and electronic excitation free energies. This is analogous to procedures that have been used to reduce shock Hugoniot data and derive room temperature standards, but the present analysis gives a strong physical foundation for the volume dependence of $\gamma$, allowing confidence in the results at higher compression. We are not aware of any evidence of solid-solid phase transitions in $\mathrm{Au}$, and we consider only the fcc solid. Recent calculations 16 indicate a transition to the hcp structure for $V / V_{0} \lesssim 0.6$, and we do not attempt to extend our solid EOS beyond this density.

This paper is arranged as follows. First we discuss lattice dynamics and its connection to the EOS. We emphasize the importance of the classical limit for defining the ion motion contribution to $\gamma$. We describe our procedures for calculating phonon frequencies and interpolating to the whole Brillouin zone. Next we present our results for elastic and phonon properties of $\mathrm{Au}$, and our analysis to obtain $\gamma^{\text {ion }}(V)$. We then present the complete EOS for solid Au by giving a set of closed form expressions for the Helmholtz free energy with parameters. The resulting room temperature isotherm is compared with various proposed standards. Next we discuss shock melting and its effect on the Hugoniot within an approximate model, and finally give our conclusions. 


\section{LATTICE DYNAMICS AND THE EQUATION OF STATE}

We write the Helmholtz free energy as

$$
F(V, T)=\phi_{0}(V)+F^{\mathrm{ion}}(V, T)+F^{\mathrm{el}}(V, T)
$$

where $\phi_{0}$ is the static lattice energy, $F^{\text {ion }}$ is the ion motion free energy, and $F^{\mathrm{el}}$ is the electronic excitation free energy. In our applications, $\phi_{0}$ gives the largest contribution to the pressure. The ion motion free energy gives the dominant temperature dependence of $P=-(\partial F / \partial V)_{T}$. The electronic excitation term $F^{\mathrm{el}}$ is generally a small correction to the solid EOS, becoming important for the liquid Hugoniot at several hundred GPa.

It is, therefore, imperative to have an accurate $F^{\text {ion }}$ for calculating the temperature dependence of $P$. The quasi-harmonic approximation has been found to have small errors in many cases 17 We have carried out Monte Carlo simulations using an embedded atom mode $1^{18}$ of $\mathrm{Cu}$ to investigate the importance of anharmonic corrections to the ion free energy. Here we define the anharmonic free energy to be the difference between the true ion free energy and the quasi-harmonic approximation, noting that the term has been used differently ${ }^{19}$ by other authors ${ }^{20}$ We intend to publish details of our $\mathrm{Cu}$ simulations elsewhere. In summary, we find that up to $300 \mathrm{GPa}$ on the melting curve, $\left|P^{\mathrm{anh}}\right|<0.85 \mathrm{GPa}$, and is never more than $3 \%$ of the thermal pressure $P(V, T)-P(V, 0)$, a very small correction to the total pressure. Given the similarity of the bonding in $\mathrm{Cu}$ and $\mathrm{Au}$, we expect these results to be relevant for Au also. Thus, in what follows we neglect anharmonicity and use the quasi-harmonic approximation for $F^{\text {ion }}$,

$$
F^{\text {ion }}(V, T)=\int_{0}^{\infty} d \omega g(\omega)\left[\frac{1}{2} \hbar \omega+k_{B} T \ln \left(1-e^{-\hbar \omega / k_{B} T}\right)\right],
$$

where we have introduced $g(\omega)=\frac{1}{3 N} \sum_{\mathbf{k}} \delta\left(\omega-\omega_{\mathbf{k}}\right)$, the normalized phonon density of states. The phonon frequencies $\omega_{\mathbf{k}}$ are functions of volume only, and the sum is over the $3 N$ normal modes of the crystal.

Application of Eq. (2) requires the full phonon density of states $g(\omega)$ at all volumes. While in principle this information is available from our calculations, in practice the classical limit dominates our EOS, allowing for a substantial simplification. In the classical limit, which is the leading term in the high temperature expansion of Eq. (2), the free energy is given by

$$
F^{\mathrm{cl}}(V, T)=3 N k_{B} T \ln \left(\frac{e^{-1 / 3} \hbar \omega_{0}}{k_{B} T}\right)
$$

where we have introduced the moment

$$
\omega_{0}=e^{1 / 3} \exp \left[\int_{0}^{\infty} d \omega g(\omega) \ln \omega\right] .
$$

Other moments are conventionally defined as

$$
\omega_{n}=\left[\frac{3+n}{3} \int_{0}^{\infty} d \omega g(\omega) \omega^{n}\right]^{1 / n} n \neq 0, n>-3,
$$

where the normalizations in Eqs. (4) and (5) are chosen so that for a Debye spectrum, described by

$$
g(\omega)=\frac{3}{\omega_{D}^{3}} \omega^{2} \Theta\left(\omega_{D}-\omega\right),
$$

all $\omega_{n}$ are equal to $\omega_{D}$, the Debye frequency. We subsequently give results for $v_{n}=\omega_{n} /(2 \pi)$ corresponding to the experimental convention of giving frequencies $v$ in Hertz, as opposed to angular frequencies, $\omega$.

In the classical limit, the ion pressure is linear in $T$ with $\left(\partial P^{\text {ion }} / \partial T\right)_{V}=\left(3 N k_{B} / V\right) \gamma_{0}$, where $\gamma_{0}=-\frac{d \ln \omega_{0}}{d \ln V}$, and the specific heat is constant, $C_{V}=3 N k_{B}$. The role of quantum ion motion in the EOS is illustrated in Figure 1 which shows the pressure along an isochore $V / V_{0}=0.8$. The solid curve is our full EOS, described below, and the dashed curve uses the classical limit of $F^{\text {ion }}$ at all $T$. The arrows mark room temperature and the Hugoniot temperature, which is $1340 \mathrm{~K}$ at the given density. The melting temperature at this density is estimated to be $4900 \mathrm{~K}$, beyond the range of the plot. It is clear that the classical limit dominates even at room temperature. The Hugoniot is well into the classical regime. At higher densities, the Hugoniot is still further above the quantum regime. The largest quantum effect on the pressure is at $T=0$, where the zero-point vibrations contribute a pressure of $0.8 \mathrm{GPa}$ at this density. The classical limit is clearly dominant for interpolating between the Hugoniot and room temperature. 


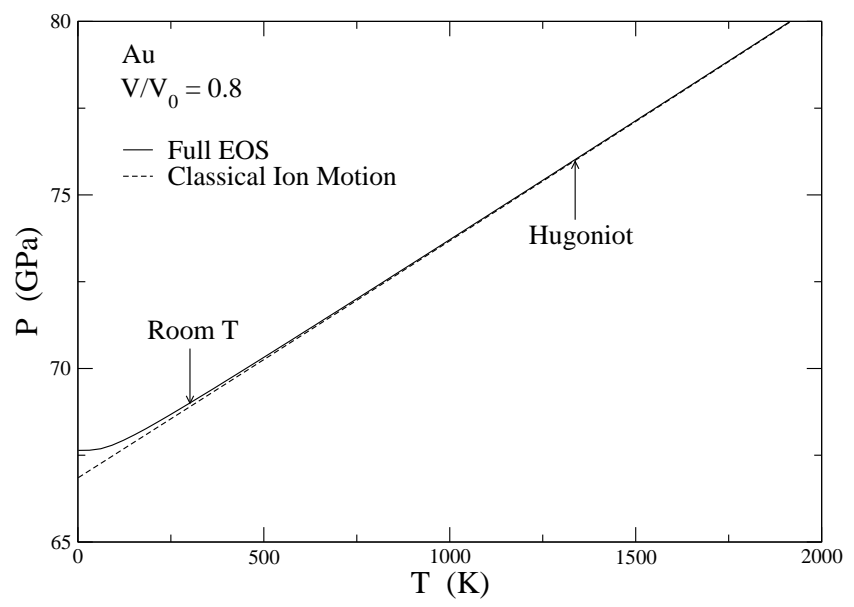

FIG. 1: Role of quantum ion motion in the EOS. Graph shows $P(T)$ along an isochore $V / V_{0}=0.8$. Solid curve is the full EOS, dashed curve uses the classical limit of the ion free energy. Hugoniot temperature at the given density is $1340 \mathrm{~K}$.

We have found that an interpolation based on the Debye model gives a very accurate approximation to the full quasi-harmonic free energy. The Debye free energy is a special case of the quasi-harmonic free energy, Eq. (2). Inserting the Debye density of states, Eq. (6), gives

$$
F^{\mathrm{D}}(V, T)=N\left[\frac{9}{8} \hbar \omega_{D}+3 k_{B} T \ln \left(1-e^{-\hbar \omega_{D} / k_{B} T}\right)-k_{B} T D\left(\hbar \omega_{D} / k_{B} T\right)\right]
$$

where

$$
D(x)=\frac{3}{x^{3}} \int_{0}^{x} d z \frac{z^{3}}{e^{z}-1} .
$$

In light of the above remarks, it is important to capture the correct classical limit. This requires that we set

$$
\omega_{D}(V)=\omega_{0}(V) .
$$

To emphasize the distinction between Eq. (9) and the standard definition of $\omega_{D}$ in terms of acoustic velocities, we refer to the Debye free energy together with Eq. (9) as the high-temperature Debye model. The high-temperature Debye model gives the same results as the full quasi-harmonic free energy in the classical regime, and in addition obeys the Nernst theorem at low $T$. We have checked that at low $T$, the error in the pressure compared to the full quasi-harmonic approximation is entirely negligible.

Thus, we can simplify the specification of the lattice vibration free energy from giving $g(\omega)$ at all $V$ to giving the single moment $\omega_{0}$ at all $V$. This is advantageous for numerical applications. It also allows us to express our full EOS in a few compact formulae so that it is generally accessible. These formulae are given below.

Calculating the moments $\omega_{n}$ requires the phonon frequencies for all $\mathbf{k}$ in the Brillouin zone. Direct ab initio calculations on a dense mesh in $\mathbf{k}$ would be quite expensive. As a result of another study ${ }^{23}$ we found that the low order moments can often be accurately obtained from short-ranged force constant models. In particular, for $\mathrm{Au}$, the moment $\omega_{0}$ is converged to less than $1 \%$ with a 2 nd neighbor $(2 \mathrm{NN})$ model. Therefore, our method is to calculate four zone boundary phonon frequencies corresponding to the transverse and longitudinal modes at the $X$ and $L$ points. These are calculated with standard frozen-phonon methods. In addition the three elastic moduli are calculated using the method described by Söderlind et al ${ }^{24}$ We fit these results to a $2 \mathrm{NN}$ force constant model, which then allows the evaluation of $\omega_{\mathbf{k}}$ for arbitrary $\mathbf{k}$ for integration over the Brillouin zone.

The electronic structure calculations used the full-potential LAPW code WIEN97,25 We used the LDA rather than the GGA, based on Boettger's finding 16 that the LDA gives a better static lattice energy than the GGA for Au. Some numerical parameters used in the calculations were, in atomic units: muffin tin radius $r_{\mathrm{MT}}=2.0$; plane wave cut-off $r_{\mathrm{MT}} k_{\max }=9.0$; cut-off for expansion of density and potential $g_{\max }=14$. For elastic modulus calculations, Brillouin zone integrals used special points corresponding to $18^{3}$ points in the full zone, with Gaussian smearing of the energies by $20 \mathrm{mRy}$. The zone boundary phonons were found to be comparatively insensitive to the k-point mesh, and smaller meshes of $10^{3}$ points were used. The $5 p, 5 d$, and $6 s$ shells were treated as valence states, and local orbital extensions ${ }^{26}$ were used in the $p$ and $d$ channels. 


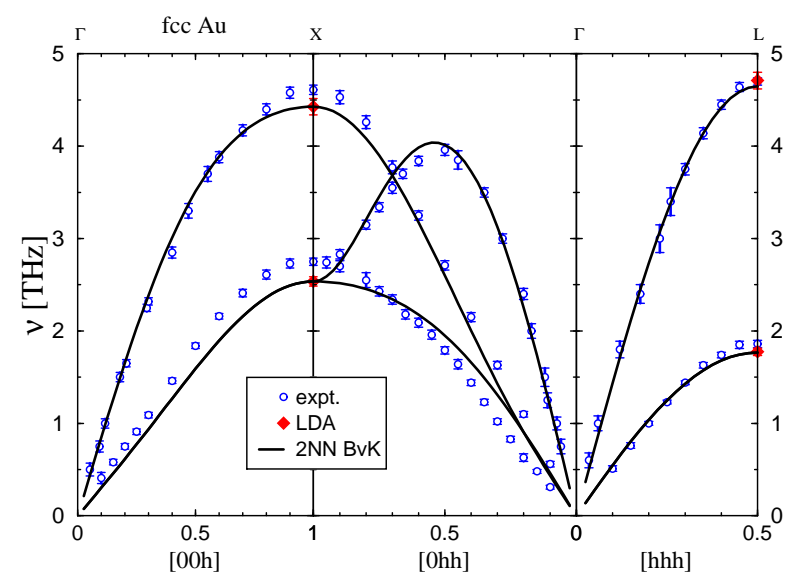

FIG. 2: (Color Online) Phonon dispersion for Au at room temperature and ambient pressure. Open circles are experimental data of Lynn et. $a l^{27}$. Filled diamonds are LDA calculations of zone boundary phonons at the $X$ and $L$ points. Solid curve is interpolation based on fit of $2 \mathrm{NN}$ force constant model to the LDA zone-boundary phonons and elastic moduli.

We used a generalized Born-von Kármán force model for our lattice-dynamical calculations, from which we were able to compute the phonon dispersions in the entire Brillouin zone. Since gold has a very simple phonon dispersion, we employed only a model with first $(1 \mathrm{NN})$ and second $(2 \mathrm{NN})$ nearest-neighbor interatomic shells of atoms. In an fcc lattice the $1 \mathrm{NN}$ and $2 \mathrm{NN}$ forces are determined by three and two independent parameters, respectively (for more details see e.g. ref.23). These five force constants were extracted by fitting simultaneously the phonon frequencies of gold at the $X$ and $L$ points of the Brillouin zone and the elastic constants $\mathrm{C}_{11}, \mathrm{C}_{12}$, and $\mathrm{C}_{44}$ near the zone center ( $\Gamma$ point). Thus we fit a total of seven independent data points. We gave equal weight to the zone boundary phonons and elastic constants in our $\chi^{2}$-fit of the phonon dispersions. Although the $2 \mathrm{NN}$ Born-von Kármán force model is too simple to reproduce all phonon frequencies within less than approximately 10\%, see Figure 2 it is sufficiently accurate to compute integrated quantities such as the phonon moments within less than approximately $3 \%$. More accurate phonon dispersions can be obtained if needed, by computing ab initio frequencies at half and quarter distances in the Brillouin zone and fitting those to a 3NN or higher-order Born-von Kármán force model, or to phonon models with interatomic pseudo-potentials.

\section{ELASTIC AND PHONON PROPERTIES}

Figure 2 shows the calculated phonon dispersion curves for $\mathrm{Au}$ at the density corresponding to ambient pressure and room temperature. The filled diamonds at the $X$ and $L$ points are the LDA frozen phonon results. These, together with the calculated elastic moduli are used to obtain the force constants. The solid curves are the interpolation to general wavevectors using the force constant model. The open circles are the experimental data of Lynn et al ${ }^{27}$ The force constant model is over-constrained, so it neither goes precisely through the calculated zone boundary frequencies, nor has it exactly the calculated elastic moduli. The shapes of the dispersion curves are simple enough for Au that they are generally well captured by the $2 \mathrm{NN}$ model.

Table 【 summarizes our results for the zone boundary phonons, elastic moduli, and moments $v_{n}=\omega_{n} / 2 \pi$, as functions of volume. The reference volume $V_{0}$ corresponds to $T=298 \mathrm{~K}$ and $P=1 \mathrm{bar}=100 \mathrm{kPa}$. For Au, $V_{0}=10.212 \mathrm{~cm}^{3} / \mathrm{mol}$ or $114.43 a_{0}^{3} /$ atom. Also shown are the experimental data at room temperature ${ }^{27.28}$ No attempt is made to account for the temperature dependence beyond comparing at the correct volume. There is generally good agreement between the calculated and experimental quantities. The main exception is $C_{44}$, which is calculated substantially lower than measured. Our calculation is in better agreement with an earlier LDA calculation ${ }^{24}$ The main result of the present calculation is the value of $v_{0}$, which is within $3 \%$ of the measurement. The ratio $v_{1} / v_{0}$ is 1 for a Debye spectrum. Our calculations give $v_{1} / v_{0}=1.03$ at ambient density and 1.04 at twofold compression, so, by this measure, the departure from a Debye spectrum is small and nearly constant with volume.

Measurements of elastic moduli were reported by Duffy et al ${ }^{29}$ to $P=37 \mathrm{GPa}$, and ab initio calculations by Tsuchiya and Kawamura ${ }^{30}$ to $100 \mathrm{GPa}$. Figure 3 shows these results along with our calculations. Our volumes are converted to room temperature pressures using the EOS described below. The highest density in table $\square$ corresponds to $P \approx 780 \mathrm{GPa}$, and the graph is restricted to lower pressures to highlight the comparison with these other works. Our calculations of $C_{11}$ and $C_{12}$ are in good agreement with Tsuchiya and Kawamura, while our $C_{44}$ is systematically lower, in somewhat better agreement with the experiments. Our $C_{11}$ is in good agreement with the experiments while $C_{12}$ is slightly high. It should be noted that the experimental $C_{i j}$ depend on a model parameter $\alpha$ used in the analysis ${ }^{29}$ The plotted points correspond to $\alpha=1$. It is encouraging 
TABLE I: Calculated phonon and elastic properties of Au. $V_{0}$ is the volume at $298 \mathrm{~K}$ and atmospheric pressure and is $10.212 \mathrm{~cm} 3 / \mathrm{mol}^{2}$ for Au. Frequencies $v$ given in $\mathrm{THz}$ and elastic moduli given in $\mathrm{GPa}$.

\begin{tabular}{|c|c|c|c|c|c|c|c|c|c|c|}
\hline$V / V_{0}$ & $v_{X t}$ & $v_{X l}$ & $\mathrm{v}_{L t}$ & $\mathrm{v}_{L l}$ & $C^{\prime}$ & $C_{44}$ & $B$ & $v_{0}$ & $v_{1}$ & $v_{2}$ \\
\hline 1.1 & 1.76 & 3.17 & 1.33 & 3.37 & 9.2 & 11.1 & 91.0 & 2.53 & 2.60 & 2.68 \\
\hline 1.0 & 2.54 & 4.43 & 1.77 & 4.71 & 13.8 & 27.4 & 172.0 & 3.53 & 3.64 & 3.75 \\
\hline 1.0/Expt. & 2.75 & 4.61 & 1.86 & 4.70 & 14.6 & 41.5 & 167. & 3.65 & 3.75 & 3.86 \\
\hline 0.9 & 3.45 & 5.91 & 2.30 & 6.32 & 20.3 & 55.0 & 304.4 & 4.71 & 4.86 & 5.00 \\
\hline 0.8 & 4.55 & 7.71 & 2.95 & 8.40 & 26.8 & 112.3 & 527.3 & 6.16 & 6.35 & 6.54 \\
\hline 0.7 & 5.94 & 10.05 & 3.76 & 11.19 & 37.4 & 221.7 & 928.3 & 8.03 & 8.30 & 8.57 \\
\hline 0.6 & 7.76 & 13.18 & 4.78 & 15.23 & 67.0 & 443.5 & 1663.8 & 10.55 & 10.93 & 11.30 \\
\hline 0.5 & 10.27 & 17.63 & 6.10 & 21.62 & 135.4 & 871.0 & 3097.4 & 13.99 & 14.58 & 15.13 \\
\hline
\end{tabular}

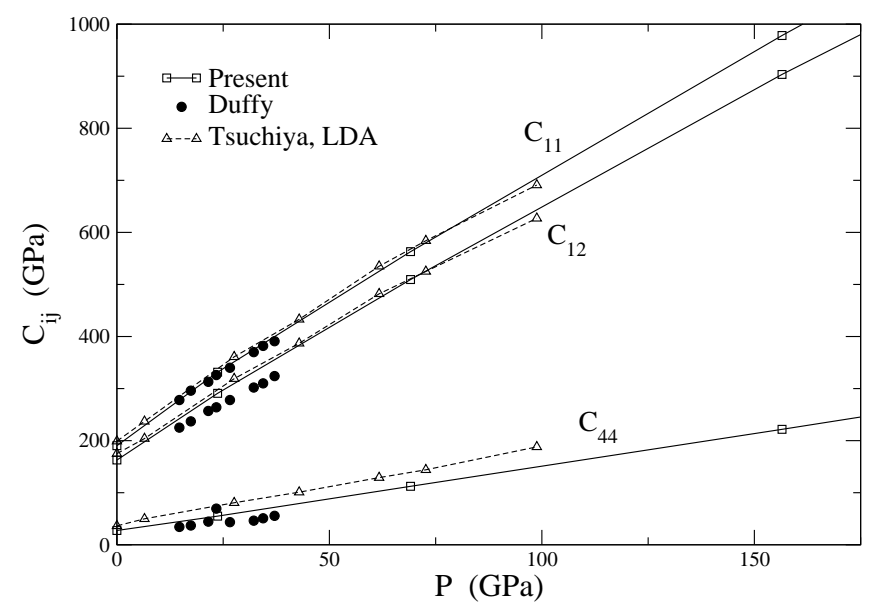

FIG. 3: Pressure dependence of the elastic moduli of Au. Open squares are present LDA calculations with pressures from our EOS. Solid circles are experimental data from Duffy et al $^{29}$. Open triangles are previous LDA calculations by Tsuchiya et al ${ }^{30}$

that our calculations give the correct trends for the pressure dependence of $C_{i j}$.

Having determined the moments $v_{n}$ as functions of volume, we need to interpolate and differentiate them to obtain thermodynamic functions. To do this, we assumed the following functional form for $\gamma_{n}(V)$, which has been used in creating many wide ranging equations of state,, 31

$$
\gamma_{n}(V)=\gamma^{\infty}+A_{n}\left(\frac{V}{V_{0}}\right)+B_{n}\left(\frac{V}{V_{0}}\right)^{2}
$$

where $\gamma^{\infty}$ is the infinite density limit of $\gamma$, and $A_{n}$ and $B_{n}$ are parameters. Alternatively, we can express $A_{n}$ and $B_{n}$ in terms of $q_{n}=d \ln \left(\gamma_{n}\right) / d \ln (V)$ and $\gamma_{n}\left(V_{0}\right)$ as

$$
\begin{aligned}
& A_{n}=\gamma_{n}\left(V_{0}\right)\left[2-q_{n}\left(V_{0}\right)\right]-2 \gamma^{\infty} \\
& B_{n}=\gamma_{n}\left(V_{0}\right)\left[q_{n}\left(V_{0}\right)-1\right]+\gamma^{\infty}
\end{aligned}
$$

Integrating $\gamma_{n}=-\frac{d \ln v_{n}}{d \ln V}$, we have

$$
v_{n}(V)=v_{n}\left(V_{0}\right)\left(\frac{V}{V_{0}}\right)^{-\gamma^{\infty}} \exp \left\{-A_{n}\left[\left(\frac{V}{V_{0}}\right)-1\right]-\frac{B_{n}}{2}\left[\left(\frac{V}{V_{0}}\right)^{2}-1\right]\right\} .
$$

By fitting this functional form to the calculated $v_{0}(V)$, we extract the parameters giving $\gamma_{0}(V)$. In our fits, we keep $\gamma^{\infty}$ fixed. The value $\gamma^{\infty}=2 / 3$ has been widely used, ${ }^{32}$ although arguments have been made for $\gamma^{\infty}=1 / 2,33$ For our application and density range, we have found that the quality of the fit and the resulting $\gamma_{0}(V)$ are insensitive to this choice, and we use $\gamma^{\infty}=2 / 3$. The fitting procedure is illustrated in Figure 4 where we show the fit for $v_{0}(V)$, the log moment and the resulting $\gamma_{0}(V)$. We did 


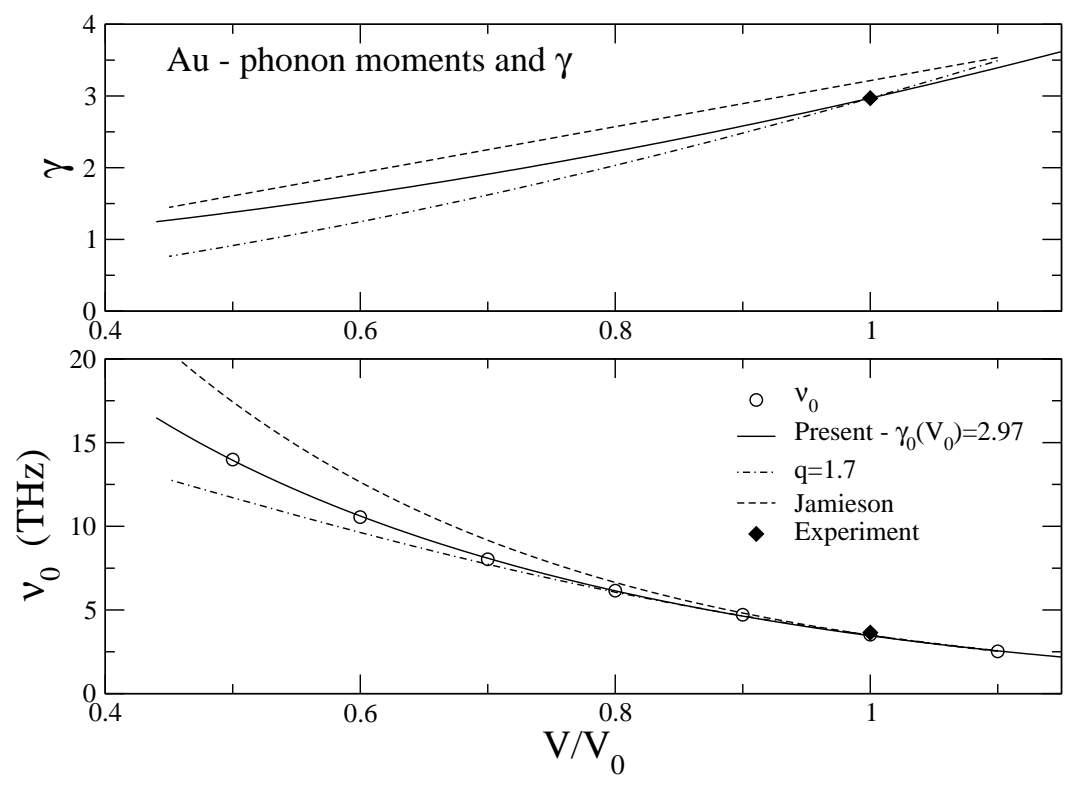

FIG. 4: Volume dependence of $v_{0}$, the $\log$ moment of the phonon frequencies, and $\gamma_{0}=-d \ln v_{0} / d \ln V$ for Au. Lower graph is $v_{0}(V)$. Open symbols are calculated moments. Upper graph is resulting $\gamma_{0}(V)$. Solid curves are present results fitting Eq. (10) to the calculated moments with the constraint $\gamma_{0}\left(V_{0}\right)=2.97$. Dash-dot curve shows the commonly used approximation $\gamma(V)=\gamma\left(V_{0}\right)\left(V / V_{0}\right)^{q}$ for $q=1.7$. 13 Dashed curve

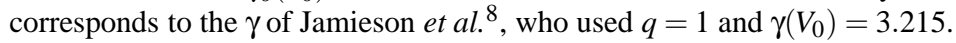

the fits with $v_{0}\left(V_{0}\right), \gamma_{0}\left(V_{0}\right)$, and $q_{0}\left(V_{0}\right)$ as free parameters, and with $\gamma_{0}\left(V_{0}\right)$ constrained to the experimental value of 2.97. (This experimental value was determined so that the thermal expansion for the subsequent EOS overlies the recommended curve of Touloukian $^{34}$ from 100 to $1200 \mathrm{~K}$.) The unconstrained fit gives $\gamma_{0}\left(V_{0}\right)=2.88$. Constraining $\gamma_{0}\left(V_{0}\right)$ increases the RMS error of the fit from $4.9 \times 10^{-2}$ to $5.7 \times 10^{-2} \mathrm{THz}$, which is not a significant increase. Also, we note that constraining $\gamma_{0}\left(V_{0}\right)$ does not change $\gamma_{0}(V)$ at smaller $V$. For this reason, we believe that the constrained fit is the best overall approximation for $\gamma^{\text {ion }}(V)$ up to twofold compression. This is shown as the solid curve in Figure 4 The corresponding parameters are $v_{0}\left(V_{0}\right)=3.46 \mathrm{THz}$, $\gamma_{0}\left(V_{0}\right)=2.97, q_{0}\left(V_{0}\right)=1.37$. We do not show the unconstrained fit in Figure 4 because it is too close to the constrained fit. The dot-dashed curves correspond to $q=$ const, $\gamma(V)=\gamma\left(V_{0}\right)\left(V / V_{0}\right)^{q}$. The value $q=1$ gives a $\gamma$ that is too high over this density range, while $q=1.7 \underline{13}^{13}$ is too low. Jamieson et al.$\underline{\underline{8}}$ used $q=1$ and an ambient value $\gamma\left(V_{0}\right)=3.215$, which results in the dashed curve. We believe that their $\gamma$ is too high at all volumes considered here. Any constant value of $q$ will lead to a $\gamma$ that is too small at very high compression.

\section{EQUATION OF STATE}

The complete EOS is determined when the Helmholtz free energy $F$ is given as a function of volume and temperature. In this section we describe our form for $F$ and give the numerical parameters for fcc Au. First we write the free energy as in Eq. (1) as the sum of the static lattice energy $\phi_{0}$, an ion motion free energy $F^{\text {ion }}$, and an electronic excitation term $F^{\mathrm{el}}$. For the ion motion free energy we use the quasi-harmonic approximation, and further specialize to the high temperature Debye model. Thus we take the ion free energy to be given by Eqs. (7) and (8), where we identify $\omega_{D}$ with the log moment $\omega_{0}$ of the phonon frequencies. As discussed in section II, this gives the correct classical limit, and leads to extremely small errors at low $T$ compared to the full phonon spectrum. This way we are able to give a closed form expression for $F^{\text {ion }}$, which is very convenient in numerical applications. The volume dependence of $\omega_{0}$ is that given in Eq. (12) with parameters given above. For the final EOS we have replaced the fitted value of $\omega_{0}\left(V_{0}\right)$ with the experimental one. This is essentially a small shift in the absolute entropy, which has negligible effect on the results. 
Electronic excitations give a small contribution to the thermodynamics, which we approximate by

$$
F^{\mathrm{el}}(V, T)=-\frac{1}{2} N \Gamma(V) T^{2}
$$

The Sommerfeld coefficient $\Gamma$ is proportional to the electronic density of states at the Fermi energy, $\Gamma=\left(\pi^{2} / 3\right) k_{B}^{2} g\left(\varepsilon_{f}\right)$. Our calculations show that $d \ln g\left(\varepsilon_{f}\right) / d \ln V \approx 0.76$ over the range of densities considered, so we take

$$
\Gamma(V)=\Gamma\left(V_{0}\right)\left(V / V_{0}\right)^{\kappa}
$$

with $\kappa=0.76$. The calculations give $\Gamma\left(V_{0}\right)=6.7 \times 10^{-4} \mathrm{~J} / \mathrm{mol} \mathrm{K}^{2}$, while the measured value from the low-temperature specific heat is ${ }^{35} \Gamma\left(V_{0}\right)=7.28 \times 10^{-4} \mathrm{~J} / \mathrm{mol} \mathrm{K}^{2}$. The measured low-temperature specific heat coefficient $\Gamma_{\text {expt }}$ is expected to be enhanced with respect to the bare value $\Gamma$ as a result of electron-phonon interactions by a factor $(1+\lambda)$, where $\lambda$ is the dimensionless electron-phonon mass enhancement parameter. The value for gold is $\lambda \approx 0.05-0.15, \frac{36}{3}$ which agrees with our calculated $\Gamma / \Gamma_{\text {expt }}=1.09$. At high temperatures, $k_{B} T>\hbar \omega_{0}$, the phonon mass enhancement becomes ineffective. Hence we use our calculated density of states in the remainder of this paper.

The static lattice energy is needed to complete the free energy. We adopt the functional form due to Vinet et al $l^{37}$

$$
\begin{aligned}
\phi_{0}(V) & =\frac{4 V^{*} B^{*}}{\left(B_{1}^{*}-1\right)^{2}}\left[1-(1+X) e^{X}\right] \\
X & =\frac{3}{2}\left(B_{1}^{*}-1\right)\left[\left(\frac{V}{V^{*}}\right)^{1 / 3}-1\right]
\end{aligned}
$$

which is parameterized by the volume at the minimum, $V^{*}$, the bulk modulus, $B^{*}$ and its pressure derivative, $B_{1}^{*}$. These parameters have been determined empirically by requiring that the EOS reproduce the ambient volume, and ultrasonic data for $B_{S}$ at ambient conditions ${ }^{28}$ For the latter, we adopt the value $B_{S}=173 \mathrm{GPa}$. Ultrasonic data gives values for $\left(\partial B_{S} / \partial P\right)_{T}$ from 5.2 to 6.4. Therefore, to complete the EOS, we require that it reproduce the measured slope of the Hugoniot, which we take from the fit ${ }^{4} U_{s}=3.12 \mathrm{~km} / \mathrm{s}+1.521 U_{p}$ relating the shock velocity $U_{s}$ to the particle velocity $U_{p}$. The resulting parameters give $\left(\partial B_{S} / \partial P\right)_{T}=5.49$, consistent with ultrasonic data.

The complete set of parameters for the free energy of fcc Au are summarized here. The static lattice energy is given by Eq. (15) with

$$
\begin{aligned}
V^{*} & =10.0834 \mathrm{~cm}^{3} / \mathrm{mol} \\
B^{*} & =180.0 \mathrm{GPa} \\
B_{1}^{*} & =5.55
\end{aligned}
$$

The ion motion free energy is given by Eqs. (7) and (8) with the volume dependence of $\omega_{D}$ given by Eq. (12) (with $v_{0} \rightarrow \omega_{D}$, $\gamma_{0} \rightarrow \gamma$, etc.). The parameters $A$ and $B$ are given in terms of $\gamma\left(V_{0}\right)$ and $q\left(V_{0}\right)$ by Eq. 111. The numerical values are

$$
\begin{aligned}
V_{0} & =10.212 \mathrm{~cm}^{3} / \mathrm{mol} \\
\omega_{D}\left(V_{0}\right) & =22.9 \times 10^{12} \mathrm{~s}^{-1} \\
\gamma\left(V_{0}\right) & =2.97 \\
q\left(V_{0}\right) & =1.3677 \\
\gamma^{\infty} & =2 / 3 .
\end{aligned}
$$

Finally, the electronic excitation free energy is given by Eqs. (13) and 14 with

$$
\begin{aligned}
\Gamma\left(V_{0}\right) & =6.7 \times 10^{-4} \mathrm{~J} / \mathrm{mol} \mathrm{K}^{2} \\
\kappa & =0.76 .
\end{aligned}
$$

The following calculations use these parameters for the free energy of fcc Au. Once the free energy is known, the pressure, internal energy, etc., can be evaluated. We calculate the Hugoniot by fixing a value of the volume and solving for the temperature such that the jump condition, $E-E_{0}=\frac{1}{2}\left(P+P_{0}\right)\left(V_{0}-V\right)$, is solved. Here $E_{0}, V_{0}$ and $P_{0}$ correspond to the initial state, taken to be ambient temperature and pressure.

Figure 5 shows the Hugoniot and room temperature isotherm for the present EOS. The solid symbols are the Hugoniot data ${ }^{5.6 .38}$ The dashed line corresponds to the linear fit for $U_{s}\left(U_{p}\right)$. Also shown as the open circles are the room temperature data from Heinz and Jeanloz ${ }^{13}$ taken to $70 \mathrm{GPa}$ with the ruby pressure scale. The open squares are the room temperature data of Bell 


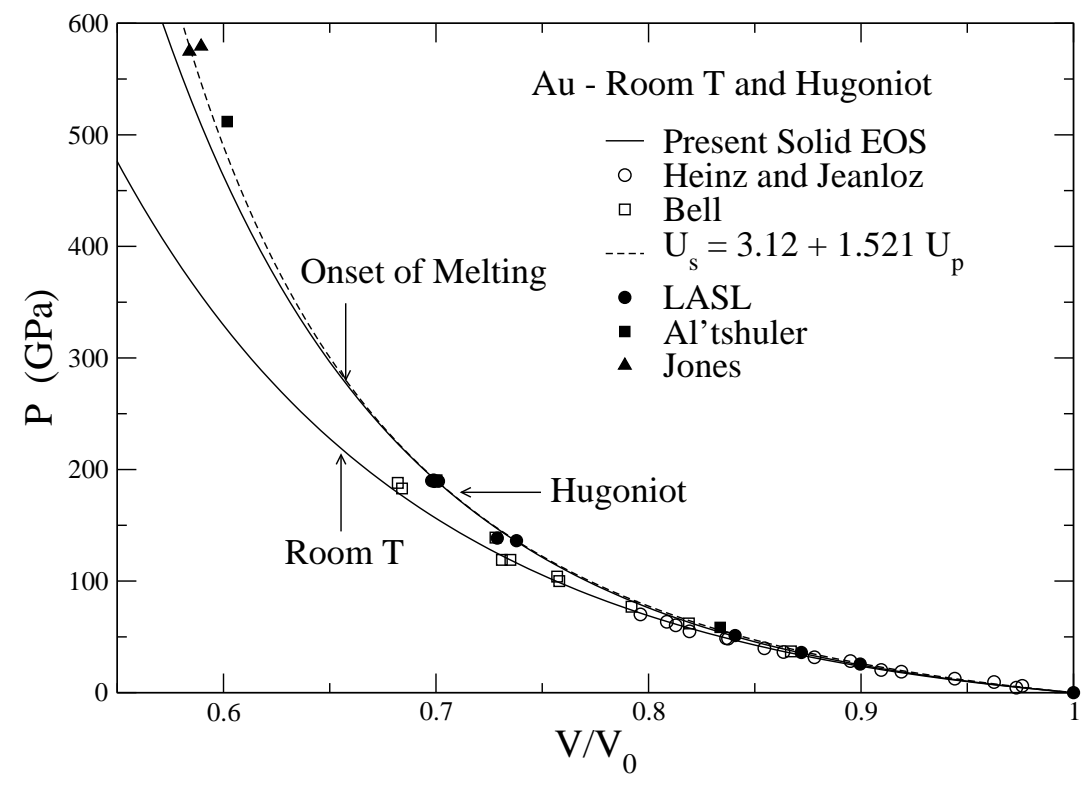

FIG. 5: Hugoniot and room temperature isotherm of Au. Solid curves are present solid EOS. Solid symbols are Hugoniot data 5,6,38 Note that there are five overlapping Hugoniot points at $V / V_{0} \approx 0.7$. Open symbols are room temperature data. ${ }^{11,13}$ For the Bell et al. data, ${ }^{11}$ pressures are from the extrapolated ruby standard. The onset of shock melting is estimated at $P \approx 280 \mathrm{GPa}$, as marked.

et al $\stackrel{11}{n}$ to $188 \mathrm{GPa}$. The pressure shown in the graph is taken from the ruby scale, which is extrapolated from a lower pressure calibration. ${ }^{9}$ Our calculations agree with these data to $3 \%$ at the highest pressure, indicating support for the extrapolation of the ruby scale.

For $V>0.656 V_{0}$, the Hugoniot lies in the solid. By matching the measured Hugoniot with our theoretically based Grüneisen parameter, we confirm the accuracy of our room temperature isotherm, and we have high confidence in our EOS to this density. Combining our calculated Hugoniot temperatures with a Lindemann melting curve, we estimate that the Hugoniot enters a solidliquid coexistence region at $V / V_{0}=0.656, P=280 \mathrm{GPa}$. A more extensive discussion of shock melting is given in the following section. There we find that explicitly accounting for melting leads to good agreement with the high pressure Hugoniot data, indicating that our solid EOS is valid to densities of $V / V_{0} \approx 0.6$.

Figure 6 shows our room temperature isotherm along with data from Heinz and Jeanloz, $\frac{13}{2}$ and Bell et al $: 11$ Also shown are some of the proposed EOS standards. Jamieson et al $\stackrel{\underline{8}}{\underline{\underline{8}}}$ used the Hugoniot as a reference, and calculated the room temperature isotherm using a Mie-Grüneisen EOS. They assumed $\gamma / V=$ const, and used $\gamma\left(V_{0}\right)=3.215$, so their $\gamma$ is always larger than ours. This results in lower room temperature pressures than ours, but by restricting their analysis to $V / V_{0}>0.775$, the impact of the assumed $\gamma$ is minimized. The Heinz and Jeanloz EOS is based on extrapolating their room temperature data, with some consideration of the shock data. Our room temperature isotherm is in good agreement with Heinz and Jeanloz to $200 \mathrm{GPa}$. The recently proposed EOS of Shim et al 14 is significantly lower than all the other standards. It is lower than the present analysis by $10 \mathrm{GPa}$ at $V / V_{0}=0.7$, corresponding to $P=156 \mathrm{GPa}$.

Figure 7 shows $P(T)$ along two isochores in comparison with various equations of state. At $V / V_{0}=0.8$, Jamieson et al. give somewhat lower $P$ at $300 \mathrm{~K}$ with a larger $d P / d T$, in keeping with their higher $\gamma$. Heinz and Jeanloz $\frac{13}{}$ are in the best overall agreement with the present pressures, with somewhat lower $d P / d T$. Shim et al ${ }^{14}$ give a somewhat higher $d P / d T$ with $P$ generally low due to their large offset at room temperature. Anderson et al ${ }^{19}$ adopted the room temperature isotherm from Heinz and Jeanloz ${ }^{13}$ Their EOS gives $d P / d T$ substantially too low at compressed volumes.

We have tabulated $P(V, T)$ in table of densities to $V / V_{0}=0.6$ and the temperatures to $5000 \mathrm{~K}$, except where such temperatures are thought to lie in the liquid phase. Boettger 16 has proposed an extension of the Au $300 \mathrm{~K}$ standard to $500 \mathrm{GPa}$ based on LDA calculations. He gives $P=344 \mathrm{GPa}$ at $V / V_{0}=0.6$ compared to $329 \mathrm{GPa}$ from our semi-empirical EOS. Given the scatter in the high pressure Hugoniot data, and our approximate treatment of melting, this $4.6 \%$ difference is probably within the uncertainties of the present analysis at this high density. 


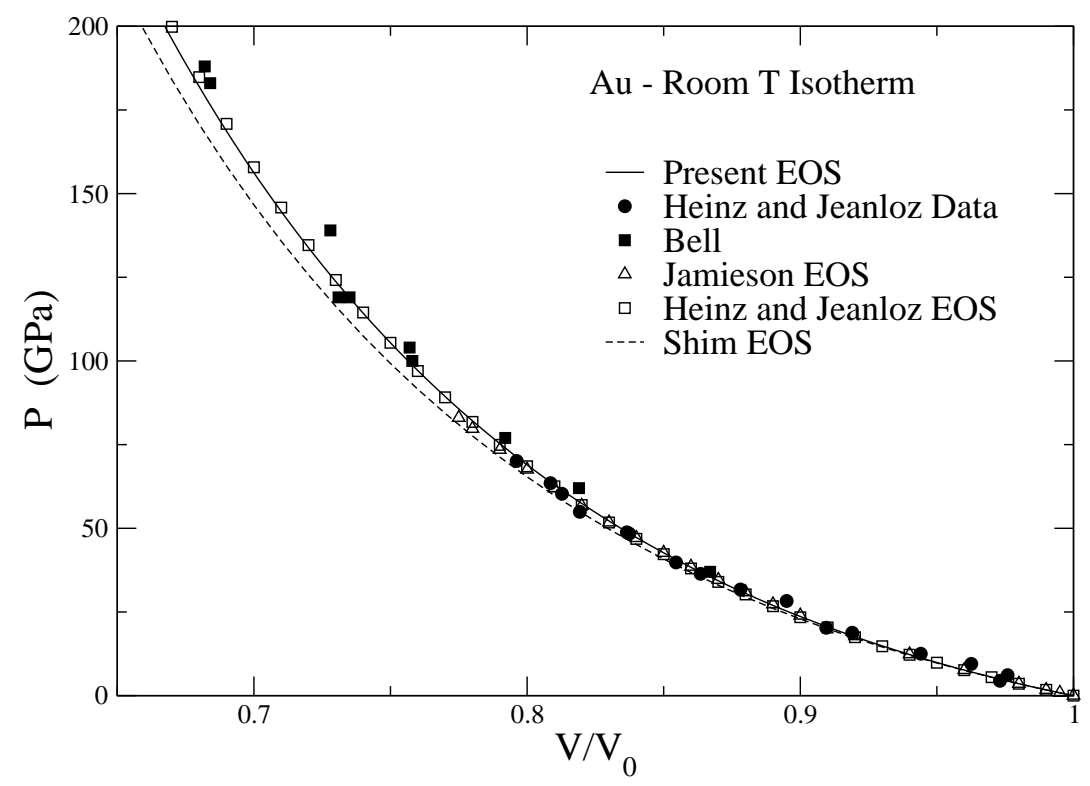

FIG. 6: Room temperature isotherm of Au. Solid curve is present EOS. Solid symbols are data 11,13 Open triangles are the EOS of Jamieson et al $\stackrel{8}{\underline{\underline{n}}}$ Open squares are EOS of Heinz and Jeanloz. ${ }^{13}$ Dashed curve is EOS of Shim et al..$^{14}$
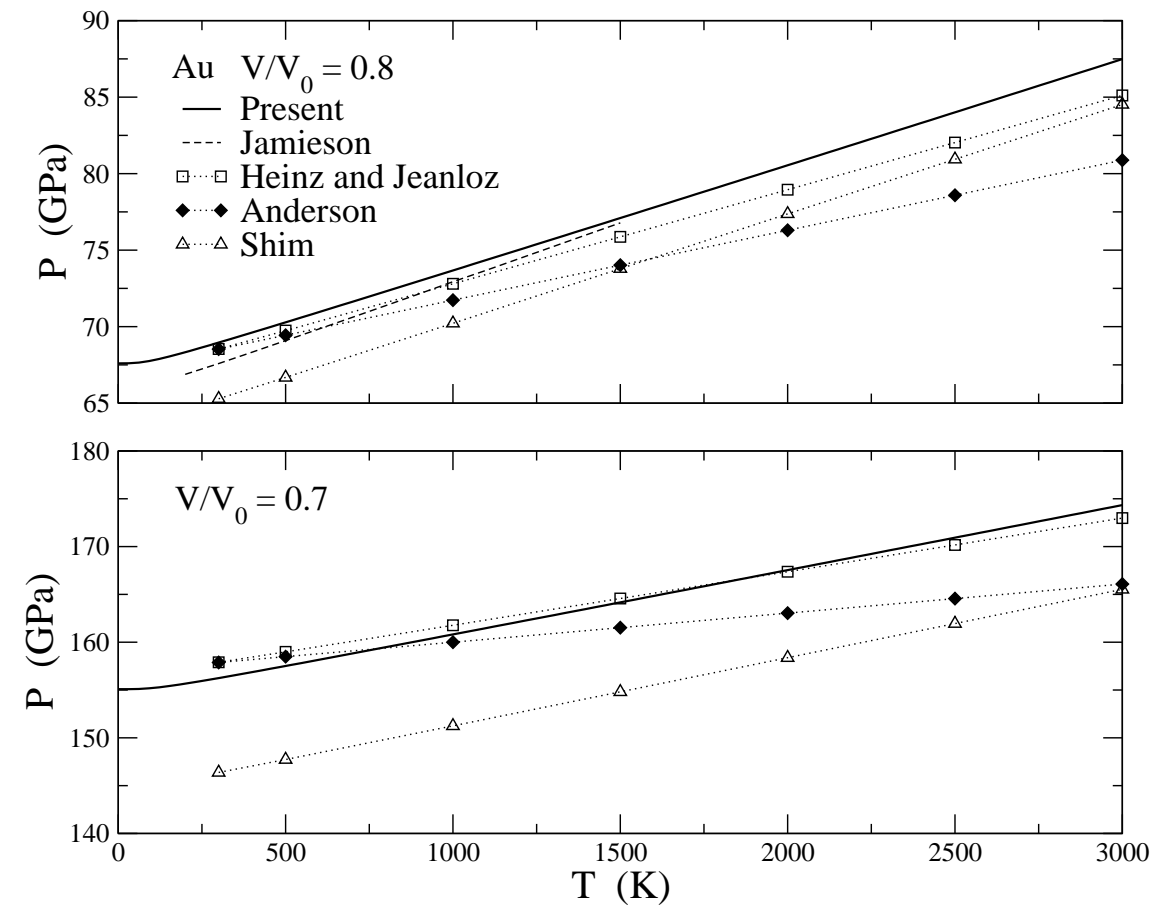

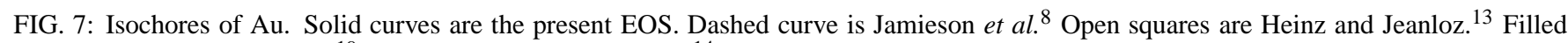
diamonds are Anderson et al.$^{19}$ Open triangles are Shim et al. 14 . 
TABLE II: Tabulated Pressures for Au. Compression $\eta=1-V / V_{300}$ where $V_{300}$ is the volume at $T=300 \mathrm{~K}$ and $P=0$. Pressures in GPa. Values in parenthesis are the first liquid states for each $\eta$, included for interpolation. Remaining liquid states left blank.

\begin{tabular}{rrrrrrrrrrrrrr}
\hline$\eta$ & $300 \mathrm{~K}$ & $500 \mathrm{~K}$ & $1000 \mathrm{~K}$ & $1500 \mathrm{~K}$ & $2000 \mathrm{~K}$ & $2500 \mathrm{~K}$ & $3000 \mathrm{~K}$ & $3500 \mathrm{~K}$ & $4000 \mathrm{~K}$ & $4500 \mathrm{~K}$ & $5000 \mathrm{~K}$ \\
\hline \hline 0.00 & 0.00 & 1.44 & 5.08 & 8.73 & $(12.40)$ & & & & & & \\
0.02 & 3.57 & 5.00 & 8.61 & 12.24 & $(15.88)$ & & & & & & \\
0.04 & 7.65 & 9.07 & 12.65 & 16.25 & 19.87 & $(23.50)$ & & & & & \\
0.06 & 12.31 & 13.71 & 17.27 & 20.85 & 24.44 & $(28.04)$ & & & & & \\
0.08 & 17.61 & 19.01 & 22.53 & 26.09 & 29.66 & 33.24 & $(36.83)$ & & & & \\
0.10 & 23.65 & 25.03 & 28.54 & 32.07 & 35.61 & 39.17 & 42.74 & $(46.33)$ & & & \\
0.12 & 30.53 & 31.90 & 35.38 & 38.88 & 42.41 & 45.94 & 49.50 & $(53.06)$ & & & \\
0.14 & 38.36 & 39.71 & 43.17 & 46.65 & 50.16 & 53.67 & 57.20 & 60.74 & $(64.30)$ & & \\
0.16 & 47.27 & 48.61 & 52.04 & 55.51 & 58.99 & 62.48 & 65.99 & 69.51 & 73.05 & $(76.60)$ & \\
0.18 & 57.41 & 58.74 & 62.15 & 65.60 & 69.06 & 72.53 & 76.02 & 79.53 & 83.04 & $(86.57)$ & \\
0.20 & 68.96 & 70.28 & 73.67 & 77.09 & 80.54 & 83.99 & 87.47 & 90.95 & 94.45 & 97.96 & $(101.49)$ \\
0.22 & 82.12 & 83.43 & 86.80 & 90.21 & 93.63 & 97.07 & 100.53 & 104.00 & 107.48 & 110.98 & 114.49 \\
0.24 & 97.13 & 98.43 & 101.78 & 105.17 & 108.58 & 112.00 & 115.44 & 118.90 & 122.37 & 125.85 & 129.34 \\
0.26 & 114.27 & 115.55 & 118.89 & 122.26 & 125.65 & 129.07 & 132.49 & 135.93 & 139.39 & 142.85 & 146.34 \\
0.28 & 133.85 & 135.12 & 138.44 & 141.80 & 145.18 & 148.58 & 151.99 & 155.42 & 158.86 & 162.32 & 165.79 \\
0.30 & 156.25 & 157.51 & 160.81 & 164.16 & 167.53 & 170.92 & 174.32 & 177.74 & 181.17 & 184.62 & 188.08 \\
0.32 & 181.92 & 183.17 & 186.46 & 189.79 & 193.16 & 196.53 & 199.93 & 203.34 & 206.76 & 210.20 & 213.65 \\
0.34 & 211.38 & 212.63 & 215.90 & 219.22 & 222.58 & 225.95 & 229.34 & 232.74 & 236.16 & 239.59 & 243.04 \\
0.36 & 245.26 & 246.49 & 249.75 & 253.07 & 256.42 & 259.79 & 263.17 & 266.57 & 269.99 & 273.41 & 276.86 \\
0.38 & 284.30 & 285.52 & 288.76 & 292.08 & 295.43 & 298.79 & 302.17 & 305.57 & 308.98 & 312.41 & 315.85 \\
0.40 & 329.37 & 330.58 & 333.82 & 337.14 & 340.48 & 343.85 & 347.23 & 350.63 & 354.04 & 357.47 & 360.91 \\
\hline
\end{tabular}

\section{MELTING AND THE HUGONIOT}

In order to investigate the effects of melting on the Hugoniot, we have constructed a two-phase model free energy. The ion free energy in the liquid is based on the assumptions that $C_{V}^{\text {ion }}=3 N k_{B}$, which is reasonable for temperatures near melting, and that $\Delta S_{V}^{\text {ion }}$, the entropy difference between solid and liquid at fixed volume is $0.8 N k_{B}$. These are empirically based model assumptions ${ }^{39}$ A statistical mechanical basis for these observations is discussed by Wallace, ${ }^{40}$ who argues for the universality of $\Delta S_{V}^{\text {ion }}$. Beyond these assumptions, we require the energy of the liquid with respect to the solid to fully determine the liquid free energy. We do this by imposing that the melting curve, obtained by equating the pressures and Gibbs free energies of liquid and solid, follow the Lindemann rule in the form

$$
\frac{T_{m}}{\omega_{0}^{2}\left(V_{s}\right) V_{s}^{2 / 3}}=\mathrm{const},
$$

where $V_{s}$ is the volume on the solidus. The electron excitation free energy is assumed to be the same in the liquid as in the solid. Application of this method to $\mathrm{Cu}$ leads to a shock melting threshold of $228 \mathrm{GPa}$, which compares well with $232 \mathrm{GPa}$ obtained by Hayes et ll $^{41}$ by analyzing sound speed data. The Au Hugoniot has been calculated for the two-phase model allowing for coexistence in the shocked state. ${ }^{42}$ Figure 8 shows the resulting Hugoniot in the pressure-volume plane. Also shown in the figure are the data points and the linear $U_{s}\left(U_{p}\right)$ fit. The dot-dashed curve is the Hugoniot for the solid only. The boundaries of the coexistence region are visible as kinks on the solid curve at 280 and $350 \mathrm{GPa}$. Above $350 \mathrm{GPa}$, the Hugoniot is in pure liquid phase. A similar anomaly is shown for $\mathrm{Al}$ in a two-phase calculation by Chisolm et al ${ }^{43}$ A significant enhancement of the

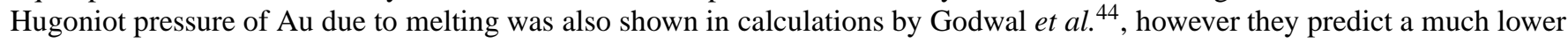
shock melting threshold than we do. Just above complete melting, the liquid Hugoniot lies above the solid by $30 \mathrm{GPa}$. The linear $U_{s}\left(U_{p}\right)$ curve goes smoothly through the melting region to intersect the liquid data.

Our two-phase EOS gives good agreement with the high pressure data, which suggests that our cold energy is valid to compressions of $V / V_{0} \approx 0.6$. Electronic excitations have practically no effect on the $P(V)$ Hugoniot in the solid phase, although they significantly affect the temperature. At higher temperatures in the liquid, they act to soften the Hugoniot by absorbing energy. Coincidentally, neglecting both electronic excitations and melting gives a Hugoniot that agrees with the linear $U_{s}\left(U_{p}\right)$ curve quite well to $650 \mathrm{GPa}$. This is an accidental cancellation of errors. Ignoring melting and electronic excitations gives a temperature that is too high by $\sim 10^{4} \mathrm{~K}$ at this pressure. This offsets the neglect of the pressure enhancement due to melting. It is not recommended to ignore either melting or electronic excitations in this high temperature regime. 


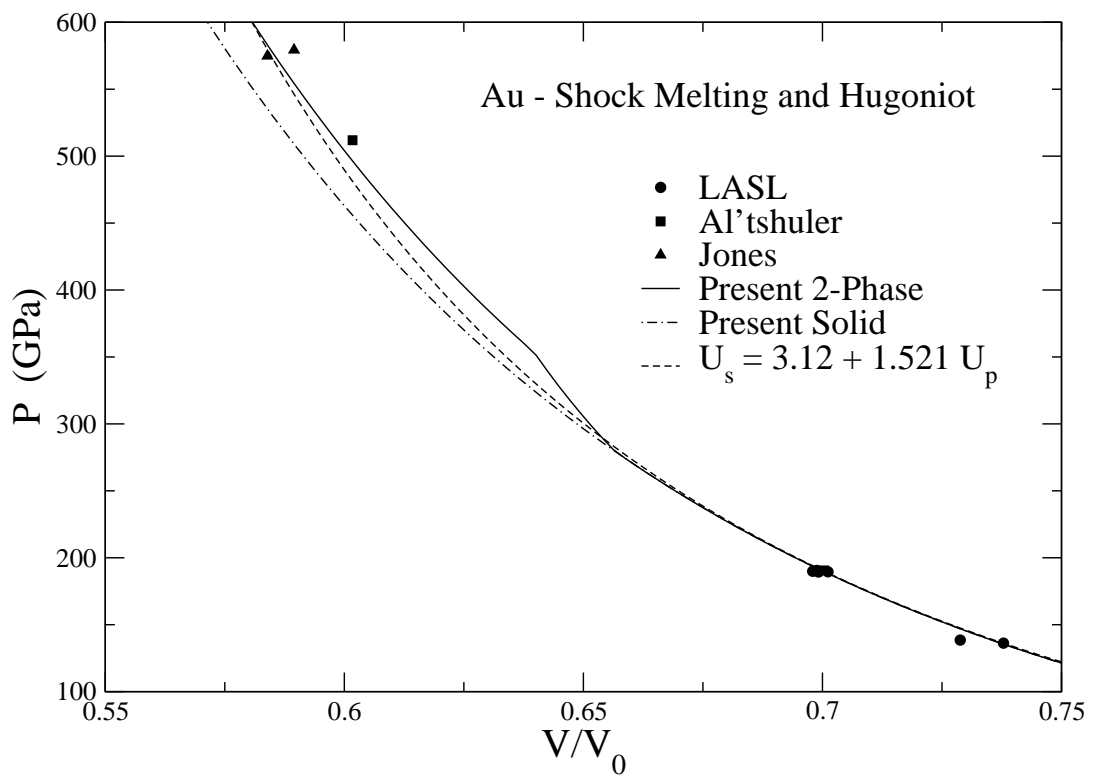

FIG. 8: Hugoniot of Au, including the effects of melting. Solid curve is present two-phase (solid and liquid) EOS. Dot-dashed curve is solid EOS only. Dashed curve is linear fit to $U_{s}\left(U_{p}\right)$ data. ${ }^{4}$ Solid symbols are measured points $, 5,6,38$

\section{CONCLUSIONS}

By combining ab initio calculations of elastic moduli and zone boundary phonons with interpolations based on a force constant model, we have calculated moments of the phonon frequencies of fcc Au to twofold compression. This allows us to calculate the associated Grüneisen parameters. In particular, we have focused on $\gamma_{0}$ which corresponds to the classical limit. We emphasize that the classical limit dominates the thermal pressure in the EOS. We find that the frequently used form $\gamma(V)=\gamma\left(V_{0}\right)\left(V / V_{0}\right)^{q}$ does not represent the volume dependence of $\gamma$ well. We have used an expansion to $2^{\text {nd }}$ order in $V$ with a physical asymptotic limit, which fits the calculated moments well and is consistent with the measured value of $\gamma$ at ambient pressure and temperature. Using the theoretical $\gamma(V)$ and electron excitation free energy, we have constructed a semi-empirical free energy for the solid, which we believe to be as accurate as possible. This free energy is given as a parameterized closed form expression and the resulting $P(V, T)$ is given in tabular form to $V / V_{0}=0.6$.

Our static lattice energy is empirical, and is verified by comparison with the measured Hugoniot, so that our EOS can be regarded as a generalization from the Hugoniot with a physically based $\gamma$. In the solid phase, electronic excitations have a small effect. This, together with the dominance of the classical limit in the vibrational free energy, means that the widely used MieGrüneisen approximation, that $(\partial P / \partial E)_{V}$ is independent of $T$, is accurate. At the highest compressions the physics affecting the Hugoniot becomes more complicated. Melting is predicted to begin at $280 \mathrm{GPa}$ and $V / V_{0}=0.656$. Complete melting is estimated to lead to a $30 \mathrm{GPa}$ increase in the Hugoniot pressure over the solid. At these densities and higher, the Hugoniot temperature is rising rapidly and electronic excitations are playing an increasing role. The highest Hugoniot data are at $580 \mathrm{GPa}$, where the temperature is calculated to be above $2 \times 10^{4} \mathrm{~K}$. The Mie-Grüneisen approximation is not expected to be valid for interpolating between the Hugoniot and room temperature at this high compression, because of the strong effects of melting and electronic excitations. These effects need to be taken explicitly into account, as has been done here.

Regarding EOS standards, our analysis gives a room temperature $P(V)$ curve that agrees well with that of Heinz and Jeanloz ${ }^{13}$ to $200 \mathrm{GPa}$. Our EOS gives $(\partial P / \partial T)_{V}$ generally somewhat larger than theirs, and we believe that in this regard our EOS is preferred. The EOS of Jamieson et al ${ }^{8}$ is based on reduction of shock data, and was originally given to $80 \mathrm{GPa}$. It gives somewhat lower pressures than ours, which is due to their use of a Grüneisen parameter that is too large at all volumes. Extrapolation of their room temperature isotherm to higher pressures ${ }^{12}$ is not recommended. Anderson et al ${ }^{19}$ adopted the room temperature isotherm of Heinz and Jeanloz ${ }^{13}$, while giving a different thermal pressure. Their EOS gives $(\partial P / \partial T)_{V}$ which is substantially too low under compression, and is not recommended for high temperatures. Simultaneous compression of Au and Pt showed ${ }^{12}$ that the Pt standard of Holmes et al ${ }^{45}$ gave a pressure higher by 15 and $20 \mathrm{GPa}$ than the Au standards of Heinz and Jeanloz ${ }^{13}$ 
and Jamieson,, 8 respectively, at a pressure of $150 \mathrm{GPa}$. While the extrapolated Jamieson isotherm is expected to be somewhat low, our analysis agrees with the room temperature isotherm of Heinz and Jeanloz, suggesting that the discrepancy between the $\mathrm{Au}$ and $\mathrm{Pt}$ pressures is due to errors in the Pt standard. Given the importance of accurate pressure standards, the status of the Pt EOS seems to warrant further investigation.

\section{Acknowledgments}

We thank J. C. Boettger, J. D. Johnson, E. D. Chisolm and S. Crockett for helpful discussions. This work was supported by the U. S. Department of Energy under Contract No. W-7405-ENG-36.

${ }^{1}$ M. H. Manghnani and T. Yagi Properties of Earth and Planetary Materials at High Pressure and Temperature. (Geophysical Union, Washington, D. C. 1998).

2 R. G. McQueen, S. P. Marsh, J. W. Taylor, J. N. Fritz, and W. J. Carter, in High Velocity Impact Phenomena, R. Kinslow Ed., (Academic, New York, 1970).

3 L. Burakovsky, C. W. Greeff, and D. L. Preston, Phys. Rev. B 67, 094107 (2003).

4 M. van Thiel, A. S. Kusubov, and A. C. Mitchell, Compendium of Shock Wave Data, Technical Report UCRL-50108, Lawrence Radiation Laboratory, Livermore, CA 1967.

5 S. P. Marsh, Ed., LASL Shock Hugoniot Data, University of California Press (Berkeley, 1980).

6 L. V. Al'tshuler, A. A. Bakanova, I. P. Dudoladov, E. A. Dynin, R. F. Trunin, B. S. Chekin, Zh. Prikl. Mekh. Tekhn. Fiz. 2, 3-34 (1981)

7 W. J. Nellis, A. C. Mitchell, and D. A. Young, J. Appl. Phys. 93, 304 (2003).

8 J. C. Jamieson, J. N. Fritz and M. H. Manghnani, in High-Pressure Research in Geophysics, edited by S. Akimoto and M. H. Manghnani (Center for Academic Publishing, Tokyo, 1982).

9 H. K. Mao, P. M. Bell, J. W. Shaner, and D. J. Steinberg, J. Appl. Phys. 49, 3276 (1978).

10 H. K. Mao, J. Xu, and P. M. Bell, J. Geo. Res.-Solid Earth and Planets, 91, 4673 (1986).

11 P. M. Bell, J. Xu, and H. K. Mao, in Shock Waves in Condensed Matter, edited by Y. M. Gupta, Plenum (New York, 1986).

12 Y. Akahama, H. Kawamura, and A. K. Singh, J. Appl. Phys. 92, 5892 (2002).

13 D. L. Heinz and R. Jeanloz, J. Appl. Phys. 55, 885 (1984).

14 S.-H. Shim, T. S. Duffy, and T. Kenichi, Earth and Planetary Science Letters 203, 729 (2002).

15 A. Khein, D. J. Singh, and C. J. Umrigar, Phys. Rev. B 51, 4105 (1995).

16 J. C. Boettger, Phys. Rev. B 67174107 (2003).

17 D. C. Wallace, Phys. Rev. E 56, 1981 (1997).

18 A. F. Voter and S. P. Chen, Mat. Res. Soc. Symp. Proc. bf 82, 175 (1987).

19 O. L. Anderson, D. G. Isaak, and S. Yamamoto, J. Appl. Phys. 65, 1534 (1989).

20 In particular, Anderson et al ${ }^{19}$ use the term anharmonicity to refer to any volume dependence of $(\partial P / \partial T)_{V}$. We impose no a priori restrictions on this quantity.

21 T. H. K. Barron, Phil. Mag. 46, 720 (1955).

22 S. P Rudin, M. D. Jones, C. W. Greeff, and R. C. Albers, Phys. Rev. B 65, 235114 (2002).

23 M. J. Graf, I.-K. Jeong, D. L. Starr, and R. J. Heffner, Phys. Rev. B 68, 064395 (2003).

24 P. Söderlind, O. Eriksson, J. M. Wills, and A. M. Boring, Phys. Rev. B 48, 5844 (1993).

25 P. Blaha, K. Schwarz, and J. Luitz, WIEN97, Vienna University of Technology 1997. (Improved and updated Unix version of the original copyrighted WIEN-code, which was published by P. Blaha, K. Schwarz, P. Sorantin, and S. B. Trickey, in Comput. Phys. Commun. 59, 399 1990).

26 D. Singh, Phys. Rev. B 43, 6388 (1991).

27 J.W. Lynn, H.G. Smith, and R.M. Nicklow, Phys. Rev. B 8 (1973) 3493.

28 G. Simmons and H. Wang, Single Crystal Elastic Constants and Calculated Aggregate Properties: a Handbook, M. I. T. Press (Cambridge 1971).

29 T. S. Duffy, G. Shen, D. L. Heinz, J. Shu, Y. Ma, H.-K. Mao, R. J. Hemley, and A. K. Singh, Phys. Rev. B, 60, 15063 (1999).

30 T. Tsuchiya and K. Kawamura, J. Chem. Phys. 116, 2121 (2002).

31 J. Abdallah, User's manual for GRIZZLY, Los Alamos National Laboratory report LA-10244-M.

32 A. V. Bushman, G. I. Kanel', A. L. Ni, and V. E. Fortov, Intense Dynamic Loading of Condensed Matter, Taylor and Francis (Washington, 1993).

33 L. Burakovsky and D. L. Preston, cond-mat/0206160

34 Y. S. Touloukian, R. K. Kirby, R. E. Taylor, and P. D. Desai, Thermal Expansion: Metallic Elements and Alloys (IFI/Plenum, New York, 1975).

35 R. Hultgren, P. D. Desai, D. T. Hawkins, M. Gleiser, K. K. Kelley, D. D. Wagman, Selected Values of the Thermodynamic Properties of the Elements (American Society for Metals, Metals Park, 1973).

36 P. B. Allen, Phys. Rev. B 36, 2920 (1987).

37 P. Vinet, J. Ferrante, J. R. Smith, and J. H. Rose, J. Phys. C: Solid State Phys. 19, L467 (1986). 
38 A. H. Jones, W. M. Isbell, and C. J. Maiden, J. Appl. Phys. 37, 3493 (1966).

39 R. Grover, J. Chem. Phys. 55, 3435 (1971).

40 D. C. Wallace, Phys. Rev. E 56, 4179 (1997).

41 D. Hayes, R. S. Hixson, and R. G. McQueen, in Shock Compression of Condensed Matter - 1999, Edited by M. D. Furnish, L. C. Chabildas, and R. S. Hixson, AIP (Melville 2000).

42 C. W. Greeff, D. R. Trinkle, and R. C. Albers, J. Appl. Phys. 90, 2221 (2001).

43 E. D. Chisolm, S. D. Crockett, and D. C. Wallace, Phys. Rev. B 68, 104103 (2003).

44 B. K. Godwal, A. Ng, and L. Dasilva, Phys. Lett. A 144, 26 (1990).

45 N. C. Holmes, J. A. Moriarty, G. R. Gathers, and W. J. Nellis, J. Appl. Phys. 66, 2962 (1989). 